\title{
Vascular endothelial growth factor polymorphisms affect gene expression and tumor aggressiveness in patients with breast cancer
}

\author{
DOONYAPAT SA-NGUANRAKSA ${ }^{1,2}$, SUWATTANEE KOOPTIWUT ${ }^{3}$, TUENJAI CHUANGSUWANICH ${ }^{4}$, \\ TAWATCHAI PONGPRUTTIPAN ${ }^{4}$, PRIDA MALASIT ${ }^{5}$ and PORNCHAI O-CHAROENRAT ${ }^{2}$ \\ ${ }^{1}$ Graduate Program in Immunology, Department of Immunology, ${ }^{2}$ Division of Head-Neck and Breast Surgery, \\ Department of Surgery; Departments of ${ }^{3}$ Physiology and ${ }^{4}$ Pathology; ${ }^{5}$ Dengue Hemorrhagic Fever Research Unit, \\ Office for Research and Development, Faculty of Medicine, Siriraj Hospital, Mahidol University, Bangkok 10700, Thailand
}

Received May 16, 2013; Accepted December 4, 2013

DOI: $10.3892 / \mathrm{mmr} .2014 .1890$

\begin{abstract}
Vascular endothelial growth factor (VEGF) is one of the key modulators of angiogenesis. The highly polymorphic promoter and 5' untranslated region of VEGF have been associated with susceptibility to and aggressiveness of several types of cancer. To examine the functional role of $V E G F$ polymorphisms at -634 and -1498 positions, $V E G F$ mRNA and protein in breast cancer tissues were analyzed by quantitative polymerase chain reaction and immunohistochemistry. A dual-luciferase assay was performed to determine promoter activity. The VEGF-634CC genotype demonstrated the highest VEGF mRNA expression. High VEGF mRNA expression was correlated with a tumor size of $>2 \mathrm{~cm}$, the presence of lymphovascular invasion and the presence of axillary nodal metastasis. The promoter containing the -1498T/-634C haplotype exhibited the highest basal promoter activity. These findings suggest that the interaction between -1498T and -634C polymorphisms increases VEGF expression and is involved in breast cancer aggressiveness.
\end{abstract}

\section{Introduction}

Vascular endothelial growth factor (VEGF) is one of the key modulators of angiogenesis. The VEGF gene is located on chromosome $6 \mathrm{p} 21.3$ and is organized as eight exons separated by seven introns $(1,2)$. Alternative exon splicing was initially shown to result in the generation of four major isoforms that were 121, 165, 189 and 206 amino acids in length (2). The $V E G F$ promoter, which spans $2.36 \mathrm{~kb}$, contains several transcription factor binding sites, including Sp1/Sp3, activating

Correspondence to: Professor Pornchai O-Charoenrat, Division of Head-Neck and Breast Surgery, Department of Surgery, Faculty of Medicine, Siriraj Hospital, Mahidol University, 2 Wanglang Road Bangkoknoi, Bangkok 10700, Thailand

E-mail: sipoc@mahidol.ac.th

Key words: angiogenesis, breast cancer, polymorphisms, vascular endothelial growth factor A, promoter activity protein (AP)-2, Egr-1, signal transducer and activator of transcription-3 and hypoxia-inducing factor-1. These transcription factor binding sites are highly conserved in mice, rats and humans. These studies indicated that the VEGF promoter is critical in the regulation of VEGF expression. Several studies showed an association between $V E G F$ polymorphisms and breast cancer susceptibility/aggressiveness, as well as levels of VEGF expression [reviewed in (3)].

Haplotype analysis showed that $-1498 \mathrm{C}$ was linked with $-634 \mathrm{G}$ (4). These two polymorphisms were associated with breast cancer susceptibility and aggressiveness (5-9). However, these polymorphisms were not located in the established transcription factor binding sites. In vitro models suggested a haplotypic effect of the polymorphic VEGF promoter on basal and stimulated promoter activity (10). In the present study, VEGF mRNA and VEGF protein expression in breast cancer tissue were determined and were correlated with various clinicopathological parameters. To verify the functional role of VEGF polymorphisms at the -634 and -1498 positions, sitedirected mutagenesis was performed to generate different $V E G F$ genotypes and to exclude other functional polymorphisms that may be in linkage disequilibrium with the polymorphisms of interest. The transcriptional activities of these polymorphisms were determined by a dual-luciferase assay.

\section{Materials and methods}

Study population. The study population was recruited from the Division of Head-Neck and Breast Surgery, Department of Surgery, Faculty of Medicine, Siriraj Hospital (Bangkok, Thailand) between 2000 and 2003. Patients with newly diagnosed breast cancer, aged $\geq 18$ years with ability to provide informed consent were included. Patients with a history of other cancers were excluded. At recruitment, informed consent was obtained, and each participant was interviewed to collect detailed information with regard to demographic characteristics. This study was approved by the Siriraj Ethics Committee on Research (Bangkok, Thailand).

Genotyping of VEGF polymorphisms. Genomic DNA was obtained from peripheral blood according to a standard method. Briefly, venous blood samples were drawn into 


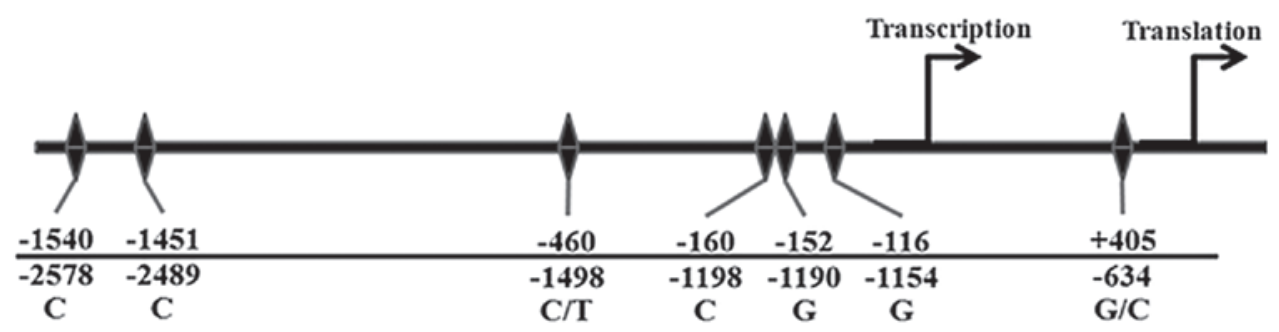

Figure 1. Haplotype of the VEGFA promoter. Transcription and translation start sites were used as references for the nomenclature of the polymorphisms in the upper line and lower lines, respectively. VEGFA, vascular endothelial growth factor A.

EDTA-containing tubes. The leukocyte cell pellet obtained from the buffy coat was resuspended with TE 20-5 solution, then digested with proteinase $\mathrm{K}$ (Promega Corporation, Madison, WI, USA) at $37^{\circ} \mathrm{C}$ overnight. DNA was isolated by the addition of phenol and chloroform-isoamyl alcohol (24:1) and centrifugation. The $V E G F-634 \mathrm{G} / \mathrm{C}$ polymorphisms were genotyped on allele refractory mutation system-polymerase chain reaction (PCR) using primers as follows: Forward, 5'-CATTGATCCGGGTTTTATCCC-3'; reverse -634G, 5'-CACTCACTTTGTCCCTGTAG-3'; reverse -634C, 5'-CAC TCACTTTGTCCCTGTAC-3'; forward control, 5'-AGA TGGTCCCTCACCTTCCT-3'; and reverse control, 5'-GTC TACCCTCCTGAGCTTGC-3'. VEGF-1498C/T polymorphisms were genotyped by PCR-restriction fragment length polymorphisms. The forward primer was 5'-TGTGCGTGT GGGGTTGAGCG-3' and the reverse primer was 5'-TACGTG CGGACAGGGCCTGA-3'. The products were digested with the Bst UI restriction enzyme (New England Biolabs, Beverly, MA, USA). -1498C products were digested and resulted in 155 and 20 bp fragments. Representative PCR products were sequenced to validate the assay.

Evaluation of VEGF expression and microvessel density (MVD) in breast cancer tissue. The levels of VEGF mRNA expression in breast cancer tissue were determined by qPCR as described previously (11). VEGF protein expression was determined by immunohistochemistry as follows: Paraffin-embedded sections were stained with a monoclonal mouse antibody to human VEGF clone VG1 (dilution, 1:100; incubation time, $1 \mathrm{~h}$; Diagnostic BioSystems, Pleasanton CA, USA) and a monoclonal antibody to CD31 (dilution, 1:300; incubation time, 16 h; Dako, Glostrup, Denmark). The immunohistochemical data was evaluated by two pathologists who had no knowledge of the patients' characteristics and/or clinical outcome. Expression of VEGF was assessed semiquantitatively using an immunohistochemical score (H score). The score was calculated by multiplying the percentage of positive carcinoma cells by the staining intensity $(0$, negative; 1 , weak; 2 , moderate; and 3, strong; as determined subjectively by the two pathologists). The average of the $\mathrm{H}$ scores from two pathologists was used. The median of the scores was used as the cutoff level to categorize tumors into low- (below the median $\mathrm{H}$ score) and high-expressing tumors (above the median $\mathrm{H}$ score), with regard to VEGF. MVD was expressed as the average number of microvessels per x 200 field. The three most intense areas of angiogenesis were identified and microvessels were counted. A single microvessel was defined as any brown immunostained endothelial cell that was separated from adjacent microvessels and other connective tissue elements. Large vessels with thick muscular walls were not counted, and the presence of a lumen was not required for scoring as a microvessel. The median of the MVD was used as the cutoff level to categorize tumors into low- and high-MVD tumors.

Construction of plasmids. pCR2.1 plasmids were purchased from Invitrogen Life Technologies (Grand Island, NY, USA). pGL3-Basic and pRL-SV40 plasmids were purchased from Promega Corporation (Madison, WI, USA). The VEGF promoter was amplified from human genomic DNA using the following primers: Forward primer, 5'-CAGGACTAGTGC ACGAATGA-3' and reverse primer, 5'-CTGTCTGTCTGT CCGTCAG-3'. The PCR reaction was conducted in a $100-\mu 1$ reaction containing 5 units of ProofStart DNA polymerase (Qiagen, Hilden, Germany), 1X PCR buffer (containing $1.5 \mathrm{mM}$ $\mathrm{MgCl}_{2}$ ), $0.2 \mathrm{mM}$ dNTPs, $0.4 \mu \mathrm{M}$ of each primer, $1 \mathrm{X} \mathrm{Q}$-solution and 800 ng genomic DNA. The 3' A-overhang was added to the purified PCR product by means of a Qiagen A-addition kit (Qiagen). This product was immediately ligated into pCR2.1 plasmid using T4 DNA ligase (Life Technologies Corporations, Grand Island, NY, USA). The ligated plasmid was transformed into Escherichia coli (E. coli) strain DH5 $\alpha$ and propagated. The constructed plasmids were fully sequenced to exclude PCR errors. The promoter was excised using HindIII and XhoI (both from New England Biolabs) prior to ligation into the pGL3-Basic vector. The promoter-reporter plasmid was resequenced to confirm correct orientation of the promoter. This plasmid was used as a template to generate other plasmids containing different polymorphisms.

Site-directed mutagenesis. Plasmids containing different polymorphisms were amplified by ProofStart DNA polymerase (Qiagen). The DNA primers were as follows: -1498Mut-C forward, 5'-GTGGGGTTGAGGGCGTTGGAGCGGGG-3' and reverse, 5'-CCCCGCTCCAACG -634Mut-C forward, 5'-GAGCAGCGAAAGCGACAGGGGC AAAGTG-3' and reverse, 5'-CACTTTGCCCCTGTCGCT TTCGCTGCTC-3'. The underlined base indicated the site of mutagenesis. The amplified products were digested by the addition of 10 units of DpnI (Stratagene, Santa Clara, CA, USA) directly to the reaction. The DpnI-treated DNA was transformed into E. coli. All plasmids were fully sequenced to exclude PCR errors and to confirm the presence of the polymorphisms. The polymorphisms in other positions are illustrated in Fig. 1.

Cell culture and DNA transfection. MCF7 breast carcinoma cells (derived from the American Type Culture Collection, 
Table I. Determination of the distribution of VEGF genotypes among breast cancer patients.

A, qPCR (total=124)

\begin{tabular}{lc}
\hline$V E G F$ gentoype & No. of patients (\%) \\
\hline-1498 & \\
TT & $65(52.42)$ \\
CT & $53(42.74)$ \\
CC & $6(4.84)$ \\
-634 & \\
GG & $57(45.97)$ \\
GC & $55(44.35)$ \\
CC & $12(9.68)$ \\
\hline
\end{tabular}

B, Immunohistochemistry (total=108)

\begin{tabular}{lc}
\hline$V E G F$ gentoype & No. of patients $(\%)$ \\
\hline-1498 & \\
TT & $58(53.70)$ \\
CT & $44(40.74)$ \\
CC & $6(5.56)$ \\
-634 & \\
GG & $47(43.52)$ \\
GC & $50(46.30)$ \\
CC & $11(10.18)$ \\
\hline
\end{tabular}

$V E G F$, vascular endothelial growth factor; qPCR, quantitative polymerase chain reaction.

Manassas, VA, USA), were cultured in Dulbecco's modified Eagle's medium with Ham's Nutrient Mixture F12 containing $10 \%$ fetal calf serum (Life Technologies, Inc., Middlesex, UK) at $37^{\circ} \mathrm{C}$ and in $5 \% \mathrm{CO}_{2}$. For transient transfection, $5 \times 10^{4}$ cells were placed in 24 -well plates and grown to $60-70 \%$ confluence. Transfection was conducted using Lipofectamine reagent Life Technologies, Inc. (Grand Island, NY, USA) according to the manufacturer's instructions. Cells were co-transfected with $V E G F$ promoter-luciferase plasmid and Renilla luciferase plasmid (pRL-SV40). After $5 \mathrm{~h}$ of incubation, fetal calf serum was added to a final concentration of $10 \%$ with or without $10^{-7} \mathrm{M}$ phorbol myristate acetate, and incubated for $16 \mathrm{~h}$ prior to evaluation of luciferase activity.

Dual-luciferase reporter assay. The cells were washed with phosphate-buffered saline and passive lysis buffer (Promega Corporation) was added to each well. The dual-luciferase reporter assay was performed according to the manufacturer's instructions. The experiments were performed in sextuplicate and repeated on three independent occasions.

Statistical analysis. The level of mRNA was calculated as the ratio of tissue sample to corresponding $\beta$-actin and then corrected as a ratio to the MDA-MB231 on the same scan. All luciferase results were expressed as a ratio to the luciferase activity of the empty vector and were normalized by Renilla
A

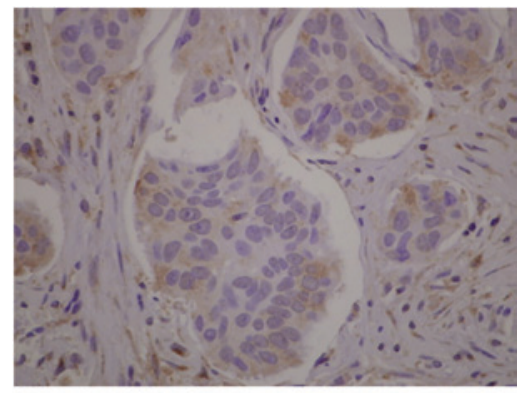

B



C

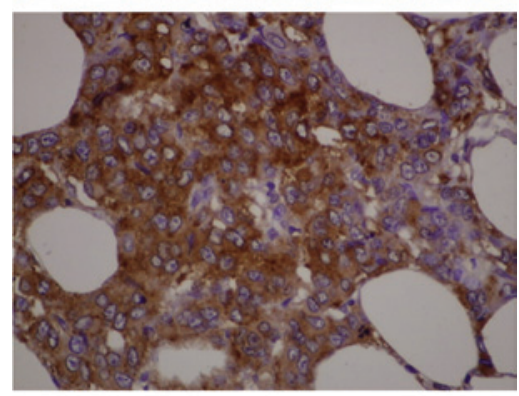

D

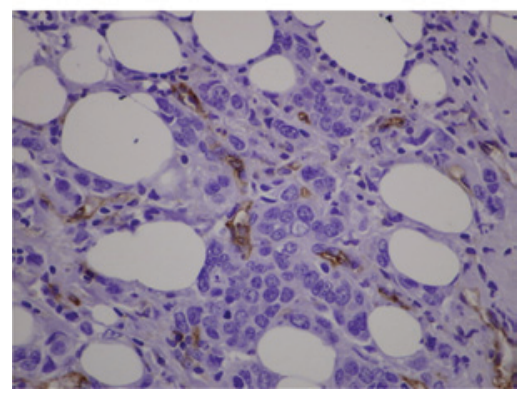

Figure 2. Representative immunohistochemical staining of VEGF and CD31. (A) 1+, Weak granular staining in the cytoplasm. (B) $2+$, Moderate cytoplasmic staining. (C) $3+$, Strong cytoplasmic staining. (D) Immunoperoxidase of CD31 demonstrates positive staining in endothelial cells. VEGF, vascular endothelial growth factor. Magnification, x200.

luciferase activity. Analysis of variance was used to evaluate the difference in mRNA expression among different genotypes and the difference in luciferase activity among haplotypes. Scheffe's post hoc test was performed to compare the difference between each pair of haplotypes. $\mathrm{P}<0.05$ was considered to indicate a statistically significant difference.

\section{Results}

Genotyping of VEGF polymorphisms. The distribution of the VEGF genotype among breast cancer patients is summarized in Table I. The characteristics of the patients were summarized in our published data (12). Due to inadequate specimens and poor tissue quality, certain breast cancer specimens were not included in the analysis. 
Table II. Normalized luciferase activity from three independent experiments.

\begin{tabular}{lcccc}
\hline & \multicolumn{3}{c}{ Normalized luciferase activity, mean (standard deviation) } \\
\cline { 2 - 5 } & $-460 \mathrm{~T}+405 \mathrm{G}$ & $-460 \mathrm{C}+405 \mathrm{G}$ & $-460 \mathrm{~T}+405 \mathrm{C}$ & $-460 \mathrm{C}+405 \mathrm{C}$ \\
\hline I & $15.12(1.55)$ & $18.56(3.43)$ & $26.05(3.83)$ & $18.04(1.60)$ \\
II & $25.98(4.05)$ & $29.21(7.18)$ & $39.41(8.85)$ & $22.91(2.79)$ \\
III & $37.32(9.69)$ & $45.03(9.78)$ & $33.14(5.98)$ & $37.79(6.18)$ \\
Average $^{\text {P-value }}$ & $25.48(10.65)$ & $30.10(12.79)$ & $3.73(12.78)$ & \\
\end{tabular}

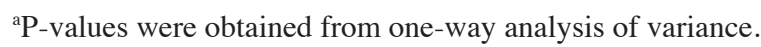

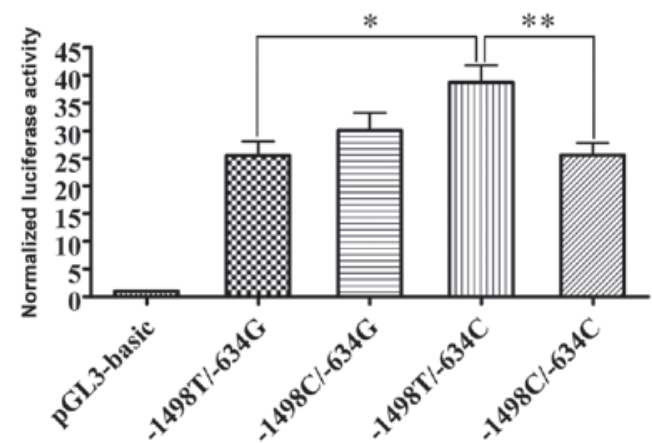

Figure 3. Luciferase activity of different $V E G F A$ promoter haplotypes in MCF-7 cells. Results are expressed as corrected relative light units \pm standard deviation. ${ }^{*} \mathrm{P}=0.014,{ }^{* *} \mathrm{P}=0.015$. VEGF $A$, vascular endothelial growth factor A.

VEGF expression in breast cancer tissue. Patients with the -634CC genotype had significantly higher VEGF mRNA in breast cancer tissue than those with $-634 \mathrm{GG}$ or -634GC genotypes $(\mathrm{P}<0.001)$. High VEGF mRNA was previously shown to be associated with a tumor size $>2 \mathrm{~cm}$ [odds ratio (OR), 2.476; 95\% confidence interval $(\mathrm{CI}), 1.047-5.858, \mathrm{P}=0.039$ ], the presence of lymphovascular invasion (OR, 2.406; 95\% CI, 1.142-5.070; $\mathrm{P}=0.021)$, and the presence of axillary nodal metastasis (OR, 2.288; 95\% CI, 1.110-4.713; $\mathrm{P}=0.025$ ) (12). Fig. 2 shows representative VEGF immunohistochemistry and staining of endothelial cells. All specimens were VEGF positive. The percentage of positive carcinoma cells ranged from 10 to $100 \%$. The intensity was expressed as weak, moderate or strong staining. The $\mathrm{H}$ score ranged from 10 to 300 . The median of the $\mathrm{H}$ score was 141.25. At this cutoff level, 54 breast carcinoma specimens were classified as exhibiting high VEGF expression. No association between $V E G F$ genotype and $\mathrm{H}$ score was observed. High VEGF H-scores were associated with higher MVD counts $(\mathrm{P}=0.021)$ and the correlation between the $\mathrm{H}$ score and MVD count was statistically significant (Pearson correlation, 0.203; $\mathrm{P}=0.035)$. No significant difference was observed between MVD and any of the four polymorphisms.

Comparison of transcriptional activity of different VEGF promoter genotypes. Comparison of the VEGF promoter and empty vector activity revealed that the $V E G F$ promoter increased basal luciferase activity of the pGL3-plasmid (Table II). The VEGF promoter bearing -1498T/-634C had significantly higher promoter activity when compared with $-1498 \mathrm{~T} /-634 \mathrm{G}$ and $-1498 \mathrm{C} /-634 \mathrm{C}(\mathrm{P}=0.014$ and 0.015 , respectively; Fig. 3). To examine mechanisms that $V E G F$ polymorphisms use to alter transcription, phorbol ester, which is known to stimulate the AP-1 site was added into the culture medium. Notably, phorbol ester increased transcriptional activity of the internal control, Renilla luciferase, more than the VEGF promoter-pGL3 Luc. This resulted in a decrease in normalized luciferase activity when compared with the basal luciferase activity of each $V E G F$ promoter genotype.

\section{Discussion}

The significant correlation between the -634CC genotype and high levels of VEGF mRNA expression in breast cancer tissues was demonstrated and was concordant with in vitro promoter activity. No correlation was identified between VEGF protein expression and $V E G F$ genotype or mRNA expression. The findings of the present study and previous studies (13-15) showed no correlation between VEGF protein expression which was determined by immunohistochemistry and VEGF genotype or mRNA expression. Failure to identify the association may be due to differences in scoring systems and the lack of reproducibility of subjective scoring in the determination of VEGF by immunohistochemistry (16). Although the levels of VEGF protein expression were not evaluated in the patients that were enrolled, several clinical trials concerning bevacizumab treatment showed satisfactory results in terms of objective response rate and progression-free survival (17-20). The majority of the patients in the present study had a VEGF intensity of $2+$ and the median proportion of positive cells was $80 \%$. This evidence suggests that almost all of the patients had relatively high VEGF levels and the expression of VEGF occurred in a dynamic manner as it varied with time.

In the current study, promoters bearing different haplotypes were generated by site-directed mutagenesis, which allowed desired loci to change and be compared, without altering the functional polymorphisms that may be in linkage disequilibrium with the loci of interest. Alteration of $-634 \mathrm{G}$ to $\mathrm{C}$ resulted in increased luciferase activity; thus, $-634 \mathrm{G} / \mathrm{C}$ polymorphisms may have a direct effect on transcriptional activity. However, no transcription factor binding motif was identified at this position (21). The transcription factor binding motif predicted by the MatInspector Online Tool found no potential transcription factor bound to this position (22). Identification of the 
transcription factor binding site using TFSEARCH version 1.3 (http://mbs.cbrc.jp/research/db/TFSEARCH.html) revealed that $-634 \mathrm{G}$ was the potential binding site for myeloid zinc finger protein 1 (MZF1), which is expressed in hematopoietic progenitor cells that are committed to myeloid lineage differentiation (23). Watson et al (4) reported that alteration from $\mathrm{G}$ to $\mathrm{C}$ diminished the potential binding capacity. However, MZF1 may not have any role in the breast cancer cells used in the current study. Transcriptional activity assessed in GI-1 human glioma cell lines and Jurkat human lymphoblastic T-lymphocyte cell lines revealed that constructions bearing -1154G/-634C haplotypes exhibited higher luciferase activity than those bearing -1154G/-634G haplotypes (24). This indicated a direct effect of the alteration from $\mathrm{G}$ to $\mathrm{C}$ at- 634 position on promoter activity, and polymorphisms at this position may regulate promoter activity at the post-transcriptional level. $\mathrm{G}$ to $\mathrm{C}$ alterations may affect the internal ribosome entry site and enhance transcription of the large VEGF isoform (395 amino acids) (25).

Stevens et al (10) constructed different haplotypes by direct amplification of genetic DNA bearing different haplotypes. This method could not ensure that the polymorphisms other than those at a specific position were identical. However, due to the high linkage disequilibrium of the VEGF promoter, this method had the advantage that the functional polymorphisms that linked with the position of interest remained linked as a block. It was reported that haplotypes containing -1198T/-1190G/634G had higher basal $V E G F$ promoter activity than haplotypes containing -1198C/-1190A/-634G. In the current study, alteration from $\mathrm{T}$ to $\mathrm{C}$ at position -1498 significantly decreased the $V E G F$ promoter activity. The interactions between these two positions contributed to the difference in promoter activity and susceptibility to/aggressiveness of breast cancer.

In conclusion, the present study demonstrated the association between mRNA expression and breast cancer aggressiveness. $V E G F$ polymorphisms altered the expression by modification of $V E G F$ promoter activity. These findings suggested that $V E G F$ polymorphisms influence growth and invasion of breast cancer cells through increased transcriptional activity and lead to increased angiogenesis. Genotyping of $V E G F$ as a potential marker for identification of the high-risk patients may therefore improve the outcome of breast cancer treatment.

\section{Acknowledgements}

This study was supported by the Research and Development Fund, Faculty of Medicine Siriraj Hospital Medical School, Mahidol University (Bangkok, Thailand).

\section{References}

1. Vincenti V, Cassano C, Rocchi M and Persico G: Assignment of the vascular endothelial growth factor gene to human chromosome 6p21.3. Circulation 93: 1493-1495, 1996.

2. Tischer E, Mitchell R, Hartman T, et al: The human gene for vascular endothelial growth factor. Multiple protein forms are encoded through alternative exon splicing. J Biol Chem 266: 11947-11954, 1991.

3. Sa-Nguanraksa D and O-Charoenrat P: The role of vascular endothelial growth factor A polymorphisms in breast cancer. Int J Mol Sci 13: 14845-14864, 2012.

4. Watson CJ, Webb NJ, Bottomley MJ and Brenchley PE: Identification of polymorphisms within the vascular endothelial growth factor (VEGF) gene: correlation with variation in VEGF protein production. Cytokine 12: 1232-1235, 2000.
5. Oliveira C, Lourenço GJ, Silva PM, et al: Polymorphisms in the 5'- and 3'-untranslated region of the VEGF gene and sporadic breast cancer risk and clinicopathologic characteristics. Tumour Biol 32: 295-300, 2011.

6. Jin Q, Hemminki K, Enquist K, et al: Vascular endothelial growth factor polymorphisms in relation to breast cancer development and prognosis. Clin Cancer Res 11: 3647-3653, 2005.

7. Balasubramanian SP, Cox A, Cross SS, Higham SE, Brown NJ and Reed MW: Influence of VEGF-A gene variation and protein levels in breast cancer susceptibility and severity. Int $\mathrm{J}$ Cancer 121: 1009-1016, 2007.

8. Schneider BP, Wang M, Radovich M, et al: Association of vascular endothelial growth factor and vascular endothelial growth factor receptor-2 genetic polymorphisms with outcome in a trial of paclitaxel compared with paclitaxel plus bevacizumab in advanced breast cancer: ECOG 2100. J Clin Oncol 26: 4672-4678, 2008.

9. Lu H, Shu XO, Cui Y, et al: Association of genetic polymorphisms in the VEGF gene with breast cancer survival. Cancer Res 65: 5015-5019, 2005.

10. Stevens A, Soden J, Brenchley PE, Ralph S and Ray DW: Haplotype analysis of the polymorphic human vascular endothelial growth factor gene promoter. Cancer Res 63: 812-816, 2003.

11. O-charoenrat P, Rhys-Evans P, Modjtahedi H and Eccles SA: Vascular endothelial growth factor family members are differentially regulated by c-erbB signaling in head and neck squamous carcinoma cells. Clin Exp Metastasis 18: 155-161, 2000.

12. Sa-Nguanraksa D, Chuangsuwanich T, Pongpruttipan T, et al: Vascular endothelial growth factor $-634 \mathrm{G} / \mathrm{C}$ polymorphism is associated with increased breast cancer risk and aggressiveness. Mol Med Rep 8: 1242-1250, 2013.

13. Kostopoulos I, Arapantoni-Dadioti P, Gogas H, et al: Evaluation of the prognostic value of HER-2 and VEGF in breast cancer patients participating in a randomized study with dose-dense sequential adjuvant chemotherapy. Breast Cancer Res Treat 96: 251-261, 2006.

14. Ludovini V, Sidoni A, Pistola L, et al: Evaluation of the prognostic role of vascular endothelial growth factor and microvessel density in stages I and II breast cancer patients. Breast Cancer Res Treat 81: 159-168, 2003.

15. MacConmara M, O'Hanlon DM, Kiely MJ, Connolly Y, Jeffers M and Keane FB: An evaluation of the prognostic significance of vascular endothelial growth factor in node positive primary breast carcinoma. Int J Oncol 20: 717-721, 2002.

16. Maae E, Nielsen M, Steffensen KD, Jakobsen EH, Jakobsen A and Sørensen FB: Estimation of immunohistochemical expression of VEGF in ductal carcinomas of the breast. J Histochem Cytochem 59: 750-760, 2011.

17. Miller KD, Chap LI, Holmes FA, et al: Randomized phase III trial of capecitabine compared with bevacizumab plus capecitabine in patients with previously treated metastatic breast cancer. J Clin Oncol 23: 792-799, 2005.

18. Miller K, Wang M, Gralow J, et al: Paclitaxel plus bevacizumab versus paclitaxel alone for metastatic breast cancer. N Engl J Med 357: 2666-2676, 2007.

19. Robert NJ, Diéras V, Glaspy J, et al: RIBBON-1: randomized, double-blind, placebo-controlled, phase III trial of chemotherapy with or without bevacizumab for first-line treatment of human epidermal growth factor receptor 2-negative, locally recurrent or metastatic breast cancer. J Clin Oncol 29: 1252-1260, 2011.

20. O'Shaughnessy JA and Brufsky AM: RiBBON 1 and RiBBON 2: phase III trials of bevacizumab with standard chemotherapy for metastatic breast cancer. Clin Breast Cancer 8: 370-373, 2008.

21. Pagès $G$ and Pouysségur J: Transcriptional regulation of the Vascular Endothelial Growth Factor gene - a concert of activating factors. Cardiovasc Res 65: 564-573, 2005.

22. Awata T, Inoue K, Kurihara S, et al: A common polymorphism in the 5'-untranslated region of the VEGF gene is associated with diabetic retinopathy in type 2 diabetes. Diabetes 51: 1635-1639, 2002.

23. Morris JF, Hromas R and Rauscher FJ III: Characterization of the DNA-binding properties of the myeloid zinc finger protein MZF1: two independent DNA-binding domains recognize two DNA consensus sequences with a common G-rich core. Mol Cell Biol 14: 1786-1795, 1994.

24. Awata T, Kurihara S, Takata N, et al: Functional VEGF C-634G polymorphism is associated with development of diabetic macular edema and correlated with macular retinal thickness in type 2 diabetes. Biochem Biophys Res Commun 333: 679-685, 2005.

25. Huez I, Bornes S, Bresson D, Créancier L and Prats H: New vascular endothelial growth factor isoform generated by internal ribosome entry site-driven CUG translation initiation. Mol Endocrinol 15: 2197-2210, 2001. 